\title{
Executive briefing: The Digital Object Identifier*
}

Primary publishers may see it as a way to manage their rights in digital content. Secondary publishers may see it as a way to create links between citations and full text.

Prospective users may even see it as a vast bibliographic database in the making ...

Chair:

Helen Atkins, Director of Database Development-Institute for Scientific Information

Speakers:

Ed Pentz-Manager Electronic Business Development, Academic Press

Albert Simmonds President, albert simmonds development

Norman Paskin Director, the International DOI Foundation

\section{The Digital Object Identifier}

Standards are back! Take the DOI, or Digital Object Identifier. At last year's conference, one speaker commented that the publishing world should stand up and take notice of the DOI "before it is DOA." It appears, though, that the DOI is in no danger of extinction.

Norman Paskin, Director of The International DOI Foundation (IDF), defines the DOI as "a unique identifier of a piece of content". It is also a system of implementation, a potential standard across all media sectors, and a unique identifier for "nameless material". Ed Pentz, Manager, Electronic Business Development, Academic Press (AP), says that the DOI system will make linking between primary and secondary publishers easier, more efficient, and more scalable.

Why add DOI metadata? Paskin offers four reasons: (1) to enable reverse look-up; (2) to enable development of DOI services; (3) for cost effectiveness; and (4) for quality control. He also notes two challenges to DOI implementation: local resolution and citation - how to find the DOI without a common platform.

\section{DOI registration}

The IDF is establishing Registration Agencies to assign and collect DOI metadata, says Paskin. There will be multiple agencies by sector and the agencies will feed the metadata to a handle service so that the metadata will be "made available to other services at the lowest possible cost". Other services can then build value-added services using the metadata.

\footnotetext{
*In cooperation with the National Information Standards Organization.
} 
Albert Simmonds, President of albert simmonds development, reports that ISBN International (a community of interest) is currently in negotiations with IDF to be the worldwide registration agency for the DOI network. The DOI Registration Agency will: (1) process applications for any entity that wants a DOI; (2) create and maintain applicant data; (3) assign the prefixes to the individual applicants and provide usage information to the prefix holders; (4) validate the DOIs themselves; (5) send the DOIs to CNRI (the handle system); (6) process DOI metadata; (7) manage links between the DOI and its URL; and (8) promote the system.

If ISBN International is named the registration agency by IDF, the three largest ISBN agencies - the US, UK, and German agencies - will be covering the entire world until other ISBN agencies are ready to take on the registration processing of applications.

"We are here to promote the widest possible use of the DOIs and their associated metadata", says Paskin. He explains that the DOI development work is not a business. Rather it is geared to provide an enabling technology/standard working in conjunction with other systems such as the Interoperability of Data in E-Commerce Systems (INDECS), URNs, Resource Description Framework (RDF), and other metadata activities.

\section{Common kernel metadata}

The sum of the IDF's work will be to arrive at a "simple, small metadata set for each market sector/genre that overlaps into a common kernel metadata that can be shared by every DOI and will ensure interoperability with standards". In the next few weeks, the IDF will take a look at reference linking as the first real application of the DOI and begin stepping up the registration process.

The DOI is being used at Academic Press (AP), says Ed Pentz, who is also Chair of the NISO DOI Syntax Committee. This committee has just issued a DOI draft standard to the DOI Discussion Group for comment. One major committee decision was to have a "dumb number" rather than an identifier code that would add meaning or intelligence in terms of the DOI system. The dumb number will require, however, intelligent metadata at registration. The committee also decided to use Unicode and the UTF-8 Character Set for the underlying handle system and the XML specification. (This has little impact on those using ASCII in their DOI strings.)

AP primarily uses the DOI in journals for linking to its online content. "We've assigned over 10,000 DOIs to articles, and we register DOIs on a weekly basis, moving to daily registration soon." AP uses its internal article ID (the unique number that AP assigns when the article is accepted) for the DOI.

AP includes the DOI in online displays and in print, as well as in the bibliographic data that it sends to others for use in linking to its content. And, AP is developing a basic look-up service for its own DOIs. $\mathrm{AP}$ is also assigning DOIs to other types of online content, such as the Encyclopedia of Immunology. On the metadata front, AP has taken its own core set of reference metadata and put it into XML for access to DOIs.

The DOI is also a part of AP's overall gateway system: IDEAL $囚$ Links. The system gives AP the flexibility to offer "simple standardized links that can be automatically created for linking into IDEAL".

To enjoy success, the DOI needs to be an open, public identifier as ubiquitous as the ISBN and ISSN, Pentz concludes. 
Web look-ups: DOI: www.doi.org (doi)

DOI: discuss-doi@doi.org (doi)

DOI: metadata@doi.org

IDEAL@LINKS: www.idealibrary.com/links/citation/0022-2836

STANDARDS IDENTIFIER PAGE: www.bisg.org 